\title{
Cervical Lymphadenitis: Etiology, Diagnosis, and Management
}

\author{
Alexander K.C. Leung, MBBS, FRCPC, FRCP(UK\&Irel), FRCPCH, \\ and H. Dele Davies, MD, MS, MHCM, FRCPC
}

\author{
Corresponding author \\ Alexander K.C. Leung, MBBS, FRCPC, FRCP(UK\&lrel), FRCPCH \\ Department of Pediatrics, University of Calgary, \\ Alberta Children's Hospital, Room 200, 233 16th Avenue NW, \\ Calgary, Alberta, Canada T2M OH5. \\ E-mail: aleung@ucalgary.ca
}

Current Infectious Disease Reports 2009, 11:183-189

Current Medicine Group LLC ISSN 1523-3847

Copyright (C) 2009 by Current Medicine Group LLC

Cervical lymphadenopathy is a common problem in children. The condition most commonly represents a transient response to a benign local or generalized infection. Acute bilateral cervical lymphadenitis is usually caused by a viral upper respiratory tract infection or streptococcal pharyngitis. Acute unilateral cervical lymphadenitis is caused by streptococcal or staphylococcal infection in $40 \%$ to $80 \%$ of cases. Common causes of subacute or chronic lymphadenitis include cat-scratch disease and mycobacterial infection. Generalized lymphadenopathy is often caused by a viral infection, and less frequently by malignancies, collagen vascular diseases, and medications. Laboratory tests are not necessary in most children with cervical lymphadenopathy. Most cases of cervical lymphadenitis are self-limited and require no treatment. The treatment of acute bacterial cervical lymphadenitis without a known primary source should provide adequate coverage for both Staphylococcus aureus and Streptococcus pyogenes.

\section{Introduction}

Enlarged cervical lymph nodes are common in children [1]. About $38 \%$ to $45 \%$ of otherwise normal children have palpable cervical lymph nodes [2]. Cervical lymphadenopathy is usually defined as cervical lymph nodal tissue measuring more than $1 \mathrm{~cm}$ in diameter [3]. Cervical lymphadenopathy most commonly represents a transient reactive response to a benign local or generalized infection, but occasionally it might herald the presence of a more serious disorder (eg, malignancy). Lymphadenitis specifically refers to lymphadenopathies that are caused by inflammatory processes $[4 \bullet \bullet]$. This article reviews the pathophysiology, etiology, differential diagnosis, clinical and laboratory evaluation, and management of children with cervical lymphadenitis.

\section{Pathophysiology}

The superficial cervical lymph nodes lie on top of the sternomastoid muscle and include the anterior group, which lies along the anterior jugular vein, and the posterior group, which lies along the external jugular vein $[4 \bullet \bullet]$. The deep cervical lymph nodes lie deep to the sternomastoid muscle along the internal jugular vein and are divided into superior and inferior groups. The superior deep nodes lie below the angle of the mandible, whereas the inferior deep nodes lie at the base of the neck.

The superficial cervical lymph nodes receive afferents from the mastoid, tissues of the neck, and the parotid (preauricular) and submaxillary nodes $[4 \bullet \bullet]$. The efferent drainage terminates in the superior deep cervical lymph nodes $[4 \bullet \bullet]$. The superior deep cervical nodes drain the palatine tonsils and the submental nodes. The lower deep cervical nodes drain the larynx, trachea, thyroid, and esophagus.

Offending organisms usually first infect the upper respiratory tract, anterior nares, oral cavity, or skin in the head and neck area before spreading to the cervical lymph nodes. The lymphatic system in the cervical area serves as a barrier to prevent further invasion and dissemination of these organisms. The nodal enlargement occurs as a result of proliferation of cells intrinsic to the node (eg, lymphocytes, plasma cells, monocytes, and histiocytes) or by infiltration of cells extrinsic to the node (eg, neutrophils). Because infections involving the head and neck areas are common in children, cervical lymphadenitis is common in this age group [5].

\section{Etiology}

Causes of cervical lymphadenopathy are listed in Table 1 [1]. The most common cause is reactive hyperplasia resulting from an infectious process, typically a viral upper respiratory tract infection [6]. Upper respiratory tract infection might be caused by rhinovirus, parainfluenza virus, influenza virus, respiratory syncytial virus, coronavirus, adenovirus, or reovirus $[1,4 \bullet \bullet]$. Other viruses associated with cervical lymphadenopathy include EpsteinBarr virus (EBV), cytomegalovirus (CMV), rubella, rubeola, 


\author{
Table 1. Causes of cervical lymphadenopathy \\ A. Infection \\ 1. Viral \\ a. Viruses causing upper respiratory infection (eg, rhino- \\ virus, parainfluenza virus, influenza virus, respiratory \\ syncytial virus, coronavirus, adenovirus, reovirus) \\ b. Epstein-Barr virus \\ c. Cytomegalovirus \\ d. Rubella \\ e. Rubeola \\ f. Varicella-zoster virus \\ g. Herpes simplex virus \\ h. Coxsackievirus \\ i. HIV
}

2. Bacterial

a. Staphylococcus aureus

b. Streptococcus pyogenes

c. Haemophilus influenzae

d. Anaerobes

e. Corynebacterium diphtheriae

f. Bartonella henselae

g. Mycobacterium tuberculosis, Mycobacterium

avium-intracellulare, Mycobacterium scrofulaceum

i. Nocardia brasiliensis

j. Pasteurella multocida

k. Treponema pallidum

3. Protozoal

a. Toxoplasma gondii

b. Leishmania species

4. Fungal

a. Candida albicans

b. Histoplasma capsulatum

c. Blastomyces dermatitides

d. Coccidioides immitis

e. Aspergillus fumigatus

B. Malignancies

1. Neuroblastoma

2. Leukemia

3. Lymphoma

4. Rhabdomyosarcoma

C. Miscellaneous

1. Kawasaki disease

2. Collagen vascular diseases

3. Serum sickness

4. Drugs

5. Postvaccination

6. Rosai-Dorfman disease

7. Kikuchi-Fujimoto disease

(Modified from Leung and Robson [5].)

varicella-zoster virus, herpes simplex virus (HSV), coxsackievirus, and HIV. Bacterial cervical lymphadenitis is usually caused by Streptococcus pyogenes (group A $\beta$-hemolytic streptococci) or Staphylococcus aureus [7]. Anaerobic bacteria can cause cervical lymphadenitis, usually in association with dental caries and periodontal disease. Group B streptococci and Haemophilus influenzae type b are less frequent causal organisms. Diphtheria is a rare cause. Bartonella henselae (cat-scratch disease), nontuberculosis mycobacteria (eg, Mycobacterium avium-intracellulare, Mycobacterium scrofulaceum), and Mycobacterium tuberculosis ("scrofula") are important causes of subacute or chronic cervical lymphadenopathy [8]. Chronic posterior cervical lymphadenitis is the most common form of acquired toxoplasmosis and is the sole presenting symptom in $50 \%$ of cases [1].

More than $25 \%$ of malignant tumors in children occur in the head and neck, and the cervical lymph nodes are the most common site [1]. During the first 6 years of life, neuroblastoma and leukemia are the most common tumors associated with cervical lymphadenopathy, followed by rhabdomyosarcoma and non-Hodgkin's lymphoma [1]. After 6 years of age, Hodgkin's lymphoma is the most common tumor associated with cervical lymphadenopathy, followed by non-Hodgkin's lymphoma and rhabdomyosarcoma.

The presence of cervical lymphadenopathy is an important diagnostic feature for Kawasaki disease. The other features include fever lasting 5 days or more, bilateral bulbar conjunctival injection, inflammatory changes in the mucosa of the oropharynx, erythema or edema of the peripheral extremities, and polymorphous rash.

Generalized lymphadenopathy might be a feature of systemic-onset juvenile rheumatoid arthritis, systemic lupus erythematosus, or serum sickness. Certain drugsnotably phenytoin, carbamazepine, hydralazine, and isoniazid—might cause generalized lymphadenopathy. Cervical lymphadenopathy has been reported after immunization with diphtheria-pertussis-tetanus, poliomyelitis, or typhoid fever vaccine [1]. Rosai-Dorfman disease is a benign form of histiocytosis characterized by generalized proliferation of sinusoidal histiocytes. The disease usually manifests in the first decade of life with massive and painless cervical lymphadenopathy, often accompanied by fever, malaise, weight loss, neutrophilic leukocytosis, elevated erythrocyte sedimentation rate, and polyclonal hypergammaglobulinemia. Kikuchi-Fujimoto disease (histocytic necrotizing lymphadenitis) is a benign cause of lymph node enlargement, usually in the posterior cervical triangle [9]. The condition primarily affects young females. Fever, nausea, weight loss, night sweats, arthralgia, myalgia, or hepatosplenomegaly might be present. The etiology of Kikuchi-Fujimoto disease is unknown, but a viral cause has been implicated [9]. Classical pathologic findings include patchy areas of necrosis in the cortical and paracortical areas of the enlarged lymph nodes and a histiocytic infiltrate [9].

\section{Differential Diagnosis}

The differential diagnosis of neck masses is different in children due to a higher incidence of infectious diseases and congenital anomalies and the relative rarity of malignancies in the pediatric age group. Cervical masses in children might be mistaken for enlarged cervical lymph nodes. In general, congenital lesions are painless and are present at birth or identified soon thereafter [10]. Clinical features that may help distinguish the various conditions from cervical lymphadenopathy are as follows. 


\section{Mumps}

The swelling of mumps parotitis crosses the angle of the jaw. On the other hand, cervical lymph nodes are usually below the mandible [1].

\section{Thyroglossal cyst}

A thyroglossal cyst is a mass that can be distinguished by its midline location between the hyoid bone and suprasternal notch and the upward movement of the cyst when the child swallows or sticks out his or her tongue.

\section{Branchial cleft cyst}

A branchial cleft cyst is a smooth and fluctuant mass located along the lower anterior border of the sternomastoid muscle.

\section{Sternocleidomastoid tumor}

A sternocleidomastoid tumor is a hard, spindle-shaped mass in the sternocleidomastoid muscle possibly resulting from perinatal hemorrhage into the muscle with subsequent healing by fibrosis [1]. The tumor can be moved from side to side but not upward or downward. Torticollis is usually present.

\section{Cervical ribs}

Cervical ribs are orthopedic anomalies that are usually bilateral, hard, and immovable. Diagnosis is established with a radiograph of the neck.

\section{Cystic hygroma}

A cystic hygroma is a multiloculated, endothelial-lined cyst that is diffuse, soft, and compressible, contains lymphatic fluid, and typically transilluminates brilliantly.

\section{Hemangioma}

A hemangioma is a congenital vascular anomaly that often is present at birth or appears shortly thereafter. The mass is usually red or bluish.

\section{Laryngocele}

A laryngocele is a soft, cystic, compressible mass that extends out of the larynx and through the thyrohyoid membrane and becomes larger with the Valsalva maneuver. There might be associated stridor or hoarseness. A radiograph of the neck might show an air fluid level in the mass.

\section{Dermoid cyst}

A dermoid cyst is a midline cyst that contains solid and cystic components. It seldom transilluminates as brilliantly as a cystic hygroma. A radiograph might show that it contains calcifications.

\section{Clinical Evaluation}

A detailed history and a thorough physical examination are essential in the evaluation of the child with cervical lymphadenopathy.

\section{History}

Age of the child

Some organisms have a predilection for specific age groups. S. aureus and group B streptococci have a predilection for neonates; $S$. aureus, group B streptococci, and Kawasaki disease for infants; viral agents, $S$. aureus, group A $\beta$-hemolytic streptococci, and atypical mycobacteria for children from 1 to 4 years of age; and anaerobic bacteria, toxoplasmosis, cat-scratch disease, and tuberculosis for children from 5 to 15 years of age. Most children with cervical lymphadenitis are 1 to 4 years of age. The prevalence of various childhood neoplasms changes with age. In general, lymphadenopathy secondary to neoplasia

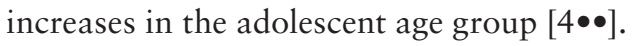

\section{Laterality and chronicity}

Acute bilateral cervical lymphadenitis is usually caused by a viral upper respiratory tract infection or pharyngitis due to $S$. pyogenes [1,11]. Acute unilateral cervical lymphadenitis is caused by $S$. pyogenes or $S$. aureus in $40 \%$ to $80 \%$ of cases $[6,12]$. The classical cervical lymphadenopathy in Kawasaki disease is usually acute and unilateral. Typically, acute suppurative lymphadenitis is caused by $S$. aureus or $S$. pyogenes [13]. Subacute or chronic cervical lymphadenitis is often caused by B. henselae, Toxoplasma gondii, EBV, CMV, nontuberculosis mycobacteria, and M. tuberculosis [1,11]. Less common causes include syphilis, Nocardia brasiliensis, and fungal infection.

\section{Associated symptoms}

Fever, sore throat, and cough suggest an upper respiratory tract infection. Fever, night sweats, and weight loss suggest lymphoma or tuberculosis. Recurrent cough and hemoptysis are indicative of tuberculosis. Unexplained fever, fatigue, and arthralgia raise the possibility of collagen vascular disease or serum sickness.

\section{Concurrent illness and past health}

Preceding tonsillitis suggests streptococcal infection. Recent facial or neck abrasion or infection suggests staphylococcal infection. Periodontal disease might indicate infections caused by anaerobic organisms. A history of cat-scratch raises the possibility of $B$. henselae infection. A history of dog bite or scratch suggests specific causative agents such as Pasteurella multocida and S. aureus. Lymphadenopathy resulting from CMV, EBV, or HIV might follow a blood transfusion. The immunization status of the child should be determined. Immunization-related lymphadenopathy might follow diphtheria-pertussis-tetanus, poliomyelitis, or typhoid fever vaccination.

\section{Drug use}

The response of cervical lymphadenopathy to specific antimicrobial therapies might help to confirm or exclude a diagnosis. Lymphadenopathy might follow the use of medications such as phenytoin and isoniazid. 


\begin{tabular}{lll}
\hline \multicolumn{2}{l}{ Table 2. Differentiation of nontuberculosis mycobacterial and Mycobacterium tuberculosis cervical lymphadenitis } \\
\hline Clinical characteristics & Nontuberculosis mycobacteria & M. tuberculosis \\
Age & 1 to 4 y & All ages (most $>5$ y) \\
Race & Predominantly white & Predominantly black or Hispanic \\
Exposure to tuberculosis & Absent & Present \\
Constitutional symptoms & Absent & Present \\
Cervical lymphadenitis & Usually solitary, in submandibular area & Usually multiple, bilateral, in posterior \\
& & cervical or supraclavicular area \\
Sinus tract formation & Approximately $10 \%$ & Rare \\
Chest radiograph & Normal $(97 \%)$ & Abnormal $(20 \%-70 \%)$ \\
Residence & Rural & Urban \\
PPD $>15$ mm of induration* & Uncommon & Usual \\
Response to antimycobacterial drugs & No & Yes \\
*PPD refers to 5 tuberculin units (5 TU) intradermal skin test. &
\end{tabular}

Exposure to infection

Exposure to a person with an upper respiratory tract infection, streptococcal pharyngitis, or tuberculosis suggests the corresponding disease. A history of recent travel should be sought.

\section{Physical examination \\ General}

Malnutrition or poor growth suggests chronic disease such as tuberculosis, malignancy, or immunodeficiency.

\section{Characteristics of the lymph tissue}

All accessible node-bearing areas should be examined to determine whether the lymphadenopathy is generalized. The nodes should be measured for future comparison [1]. Fluctuation in size of the nodes suggests a reactive process, whereas relentless increase in size indicates a serious pathology [1,14]. Tenderness, erythema, warmth, mobility, fluctuance, and consistency should be assessed.

The location of involved lymph nodes often gives clues to the entry site of the organism and should prompt a detailed examination of that site. Submandibular and submental lymphadenopathy is most often caused by an oral or dental infection, although this feature may also be seen in cat-scratch disease and non-Hodgkin's lymphoma. Acute posterior cervical lymphadenitis is classically seen in persons with rubella and infectious mononucleosis [1,11]. Supraclavicular or posterior cervical lymphadenopathy carries a much higher risk for malignancy than does anterior cervical lymphadenopathy. Cervical lymphadenopathy associated with generalized lymphadenopathy is often caused by a viral infection. Malignancies (eg, leukemia or lymphoma), collagen vascular diseases (eg, juvenile rheumatoid arthritis or systemic lupus erythematosus), and some medications are also associated with generalized lymphadenopathy.

In lymphadenopathy resulting from a viral infection, the nodes are usually bilateral and soft and are not fixed to the underlying structure. When a bacterial pathogen is present, the nodes can be either unilateral or bilateral, are usually tender, might be fluctuant, and are not fixed. The presence of erythema and warmth suggests an acute pyogenic process, and fluctuance suggests abscess formation. A "cold" abscess is characteristic of infection caused by mycobacteria, fungi, or B. henselae. In patients with tuberculosis, the nodes might be matted or fluctuant, and the overlying skin might be erythematous but is typically not warm [8]. Clinical features that help differentiate nontuberculosis mycobacterial cervical lymphadenitis from $M$. tuberculosis cervical lymphadenitis are summarized in Table $2[3,15]$. Approximately $50 \%$ of patients with lymphadenitis caused by nontuberculosis mycobacteria develop fluctuance of the lymph node and spontaneous drainage; sinus tract formation occurs in $10 \%$ of affected patients $[4 \bullet \bullet, 16 \bullet \bullet]$. In lymphadenopathy resulting from malignancy, signs of acute inflammation are absent, and the lymph nodes are hard and often fixed to the underlying tissue.

\section{Associated signs}

A thorough examination of the ears, eyes, nose, oral cavity, and throat is necessary. Acute viral cervical lymphadenitis is variably associated with fever, rhinorrhea,

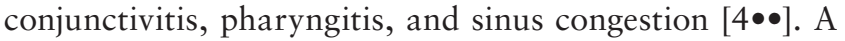
beefy red throat, exudate on the tonsils, petechiae on the hard palate, and a strawberry tongue suggest infection caused by $S$. pyogenes [1]. Unilateral facial or submandibular swelling, erythema, tenderness, fever, and irritability in an infant suggest group B streptococcal infection [13]. Diphtheria is associated with edema of the soft tissues of the neck, often described as "bull-neck" appearance. The presence of gingivostomatitis suggests infection with HSV, whereas herpangina suggests infection with coxsackievirus [11]. Rash and hepatosplenomegaly suggest EBV or CMV infection $[4 \bullet \bullet]$. The presence of pharyngitis, maculopapular rash, and splenomegaly suggest EBV infection [17]. Conjunctivitis and Koplik spots are characteristics of rubeola. The presence of pallor, petechiae, bruises, sternal 
tenderness, and hepatosplenomegaly suggests leukemia. Prolonged fever, conjunctival infection, oropharyngeal mucous membrane inflammation, peripheral edema or erythema, and a polymorphous rash are consistent with Kawasaki disease.

\section{Diagnostic Evaluation}

Laboratory tests are not necessary in most children with cervical lymphadenopathy. A complete blood cell count might help to suggest a bacterial lymphadenitis, which is often accompanied by leukocytosis with a shift to the left and toxic granulations. Atypical lymphocytosis is prominent in infectious mononucleosis [17]. Pancytopenia, leukocytosis, or the presence of blast cells suggests leukemia. The erythrocyte sedimentation rate and C-reactive protein are usually significantly elevated in persons with bacterial lymphadenitis. Blood culture should be obtained if the child appears toxic. A rapid streptococcal antigen test or a throat culture might be useful to confirm a streptococcal infection [18]. An electrocardiogram and echocardiogram are indicated if Kawasaki disease is suspected.

Skin tests for tuberculosis should be performed in patients with subacute or chronic adenitis. Chest radiography should be performed if the tuberculin skin test is positive or if an underlying chest pathology is suspected, especially in the child with chronic or generalized lymphadenopathy. Serologic tests for $B$. henselae, EBV, CMV, brucellosis, syphilis, and toxoplasmosis should be performed when indicated. If the serology is positive, the diagnosis can be established and excision biopsy can be avoided [19•].

Ultrasonography (US) is the most useful diagnostic imaging modality in the assessment of cervical lymph nodes. US may help to differentiate a solid mass from a cystic mass and to establish the presence and extent of suppuration or infiltration. High-resolution and color US can provide detailed information on the longitudinal and transverse diameter, morphology, texture, and vascularity of the lymph node $[4 \bullet \bullet, 14]$. A long-to-short axis ratio greater than 2 suggests benignity, whereas a ratio less than 2 suggests malignancy [14]. In lymphadenitis caused by an inflammatory process, the intranodal vasculature is dilated, whereas in lymphadenopathy secondary to neoplastic infiltration, the intranodal vasculature is usually distorted. Absence of an echogenic hilus and overall lymph node hyperechogenicity are suggestive of malignancy [20•]. US can also be used to guide core-needle biopsy for diagnosing the cause of cervical lymphadenopathy in patients without known malignancy and may obviate unnecessary excisional biopsy [21•]. Advantages of US include cost-effectiveness, noninvasiveness, and absence of radiation hazard. A potential drawback is its lack of absolute specificity and sensitivity in ruling out neoplastic processes as the cause of lymphadenopathy $[4 \bullet \bullet]$.

Diffusion-weighted MRI with apparent diffusion coefficient mapping can be helpful to differentiate malignant from benign lymph nodes and delineate the solid, viable part of the lymph node for biopsy [22]. The technique also allows detection of small lymphadenopathies.

Fine-needle aspiration and culture of a lymph node is a safe and reliable procedure to isolate the causative organism and to determine the appropriate antibiotic when bacterial infection is the cause [23]. Failure to improve or worsening of the patient's condition while on antibiotic treatment is an indication for fine-needle aspiration and culture $[4 \bullet \bullet]$. All aspirated material should be sent for Gram and acid-fast stain and cultures for aerobic and anaerobic bacteria, mycobacteria, and fungi $[4 \bullet \bullet, 24]$. If the Gram stain is positive, only bacterial cultures are mandatory. Polymerase chain reaction testing is a fast and useful technique for the demonstration of mycobacterial DNA fragments [15].

An excisional biopsy with microscopic examination of the lymph node might be necessary to establish the diagnosis if symptoms or signs of malignancy are present or if the lymphadenopathy persists or enlarges in spite of appropriate antibiotic therapy and the diagnosis remains in doubt [5]. The biopsy should be performed on the largest and firmest node that is palpable, and the node should be removed intact with the capsule $[1,10]$.

\section{Management}

Treatment of cervical lymphadenopathy depends on the underlying cause. Most cases are self-limited and require no treatment other than observation. This applies especially to small, soft, and mobile lymph nodes associated with upper respiratory infections, which are often viral in origin. These children require follow-up in 2 to 4 weeks.

The treatment of acute bacterial cervical lymphadenitis without a known primary infectious source should provide adequate coverage for both $S$. aureus and $S$. pyogenes, pending the results of the culture and sensitivity tests [5]. Appropriate oral antibiotics include cloxacillin, cephalexin, cefprozil, or clindamycin [6]. Children with cervical lymphadenopathy and periodontal or dental disease should be treated with clindamycin or a combination of amoxicillin and clavulanic acid, which provide coverage for anaerobic oral flora [6,25]. Referral to a pediatric dentist for treatment of the underlying periodontal or dental disease is warranted. Antimicrobial therapy may have to be modified once a causative agent is identified, depending on the clinical response of the existing treatment. Because of its proven efficacy, safety, and narrow spectrum of antimicrobial activity, penicillin remains the drug of choice for adenitis caused by $S$. pyogenes, except in patients allergic to penicillin [7]. Methicillin-resistant $S$. aureus is resistant to many kinds of antibiotics. Currently, vancomycin is the drug of choice for complicated cases, although trimethoprimsulfamethoxazole or clindamycin is often adequate for uncomplicated outpatient management [26].

In most patients, symptomatic improvement should be noted after 48 to 72 hours of therapy. Fine-needle aspiration and culture should be considered if there is no 
clinical improvement or if the patient's condition deteriorates. If the lymph nodes become fluctuant, incision and drainage should be performed. Failure of regression of lymphadenopathy after 4 to 6 weeks might be an indication for a diagnostic biopsy [12]. Indications for early excision biopsy for histology include lymph node in the supraclavicular area, lymph node larger than $3 \mathrm{~cm}$, lymph nodes in children with a history of malignancy, and clinical findings of fever, night sweats, weight loss, and hepatosplenomegaly [19•].

Toxic or immunocompromised children and those who do not tolerate, will not take, or fail to respond to oral medication should be treated with intravenous nafcillin, cefazolin, or clindamycin [6]. Oral analgesia with medication such as acetaminophen might help to relieve associated pain.

The current recommendation for the treatment of isolated cervical tuberculosis lymphadenitis is 2 months of isoniazid, rifampin, and pyrazinamide, followed by 4 months of isoniazid and rifampin by directly observed therapy for drug-susceptible M. tuberculosis [27]. If possible drug resistance is a concern, ethambutol or an aminoglycoside should be added to the initial three-drug combination until drug susceptibilities are determined, and an infectious disease specialist should be consulted [27]. Nontuberculosis mycobacterial lymphadenitis is best treated with surgical excision of all visibly infected

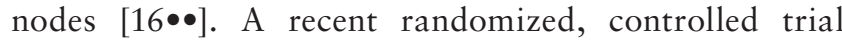
enrolled 100 children with nontuberculous cervical adenitis to receive surgical excision $(n=50)$ or antibiotic therapy with clarithromycin and rifabutin $(n=50)$ [16••]. Based on intention-to-treat analysis, the surgical cure rate was $96 \%$ versus $66 \%$ in the medical arm after 6 months. Furthermore, there were more adverse events in the medical arm. However, the major complication of surgery is permanent damage to the facial nerve, which occurred in about $2 \%$ of patients. Transient facial nerve involvement occurred in another $12 \%[16 \bullet \bullet$. Thus, careful consideration must be given to the location of the adenitis in the determination of node removal. When surgery is not feasible due to risk to the facial nerve, a two-drug antimycobacterial regimen that includes a macrolide should be considered $[6,16 \bullet \bullet, 28]$. Failure of medical therapy usually cannot be explained as a result of development of resistant organisms $[16 \bullet \bullet]$.

\section{Conclusions}

Cervical lymphadenopathy is a common and usually benign finding in children. In most cases, it is infectious in origin secondary to a viral upper respiratory tract infection. A good history and thorough physical examination are usually all that is necessary to establish a diagnosis. Most children with cervical lymphadenopathy require no specific treatment, but do need follow-up in 2 to 4 weeks. The treatment of acute bacterial cervical lymphadenitis without a known primary infectious source should provide adequate coverage for both $S$. aureus and $S$. pyogenes.

\section{Acknowledgment}

This article was published in part by Leung and Robson [1] in the Journal of Pediatric Health Care, with permission from Elsevier. It has been significantly updated for the current article.

\section{Disclosure}

No potential conflicts of interest relevant to this article were reported.

\section{References and Recommended Reading}

Papers of particular interest, published recently, have been highlighted as:

- Of importance

- Of major importance

1. Leung AK, Robson WL: Childhood cervical lymphadenopathy. J Pediatr Health Care 2004, 18:3-7.

2. Larsson LO, Bentzon MW, Berg K, et al.: Palpable lymph nodes of the neck in Swedish schoolchildren. Acta Paediatr 1994, 83:1092-1094.

3. Darville T, Jacobs RF: Lymphadenopathy, lymphadenitis and lymphangitis. In Pediatric Infectious Diseases: Principles and Practice. Edited by Jenson HB, Baltimore RS. Philadelphia: WB Saunders; 2002:610-629.

4.•• Gosche JR, Vick LV: Acute, subacute, and chronic cervical lymphadenitis in children. Semin Pediatr Surg 2006, 15:99-106.

This is an excellent article that addresses the current approaches to the diagnosis and management of cervical lymphadenitis in children.

5. Leung AK, Robson WL: Cervical lymphadenopathy in children. Can J Pediatr 1991, 3:10-17.

6. Peters TR, Edwards KM: Cervical lymphadenopathy and adenitis. Pediatr Rev 2000, 21:399-404.

7. Leung AK, Kellner JD: Group A b-hemolytic streptococcal pharyngitis in children. Adv Ther 2004, 21:277-287.

8. Spyridis P, Maltezou HC, Hantzakos A: Mycobacterial cervical lymphadenitis in children: clinical and laboratory factors of importance for differential diagnosis. Scand J Infect Dis 2001, 33:362-366.

9. Qadri F, Atkin GK, Thomas D, Das SK: Kikuchi's disease: an important cause of cervical lymphadenopathy. Clin Med 2007, 7:82-84.

10. Twist CJ, Link MP: Assessment of lymphadenopathy in children. Pediatr Clin North Am 2000, 49:1009-1025.

11. Al-Dajani N, Wootton SH: Cervical lymphadenitis, suppurative parotitis, thyroiditis, and infected cysts. Infect Dis Clin North Am 2007, 21:523-541.

12. Chesney PJ: Cervical lymphadenopathy. Pediatr Rev 1994, 15:276-284.

13. Tracy TF Jr, Muratore CS: Management of common head and neck masses. Semin Pediatr Surg 2007, 16:3-13.

14. Niedzielska G, Kotowski M, Niedzielska A, et al.: Cervical lymphadenopathy in children-incidence and diagnostic management. Int J Pediatr Otorhinolaryngol 2007, 71:51-56.

15. Bayazit YA, Bayazit N, Namidura M: Mycobacterial cervical lymphadenitis. ORL J Otorhinolaryngol Relat Spec 2004, 66:275-280.

16.• Lindeboom JA, Kuijper EJ, Bruijnesteijn van Coppenraet ES, et al.: Surgical excision versus antibiotic treatment for nontuberculous mycobacterial cervicofacial lymphadenitis in children: a multicenter, randomized, controlled trial. Clin Infect Dis 2007, 44:1057-1064.

This multicenter, randomized, controlled trial compared surgical excision versus antibiotic treatment for nontuberculous myocobacterial cervicofacial lymphadenitis in children. 
17. Leung AK, Pinto-Rojas A: Infectious mononucleosis. Consultant 2000, 40:134-136.

18. Leung AK, Newman R, Kumar A, et al.: Rapid antigen detection testing in diagnosing group A b-hemolytic streptococcal pharyngitis. Expert Rev Mol Diagn 2006, 6:761-766.

19.• Kubba H: A child with cervical lymphadenopathy. Clin Otolaryngol 2006, 31:433-434.

This excellent article offers practical guidelines on the management of childhood cervical lymphadenopathy.

20. Chan JM, Shin LK, Jeffrey RB: Ultrasonography of abnormal neck lymph nodes. Ultrasound Q 2007, 23:47-54.

This review article outlines the usefulness of ultrasonography in the assessment of cervical lymphadenopathy.

21.• Kim BM, Kim EK, Yang WI, et al.: Sonographically guided core needle biopsy of cervical lymphadenopathy in patients without known malignancy. J Utraasound Med 2007, 26:585-591.

This retrospective study showed a high yield and accuracy of sonographically guided core-needle biopsy for diagnosing the cause of cervical lymphadenopathy.
22. Abdel Razek AA, Soliman NY, Elkhamary MK, Tawfik A: Role of diffusion-weighted MR imaging in cervical lymphadenopathy. Eur Radiol 2006, 16:1468-1477.

23. Buchino JJ, Jones VF: Fine needle aspiration in the evaluation of children with lymphadenopathy. Arch Pediatr Adolesc Med 1994, 148:1327-1330.

24. Umapthy N, De R, Donaldson I: Cervical lymphadenopathy in children. Hosp Med 2003, 64:104-107.

25. Brook I, Frazier EH: Microbiology of cervical lymphadenitis in adults. Acta Otolaryngol 1998, 118:443-446.

26. Steele RW: Methicillin-resistant Staphylococcus aureus: how best to treat now? Consultant Pediatrician 2005, 4:274-276

27. American Academy of Pediatrics: Tuberculosis. In 2006 Red Book: Report of the Committee on Infectious Diseases, edn 27. Edited by Pickering LK. Elk Grove Village, IL: American Academy of Pediatrics; 2006:678-704.

28. Harza R, Robson CD, Perez-Atayde AR, et al.: Lymphadenitis due to nontuberculous mycobacteria in children: presentation and response to therapy. Clin Infect Dis 1999, 28:123-129. 